\title{
Poly-y-glutamic acid enhanced drought resistance of maize by improving photosynthesis and affecting rhizosphere microbial community
}

\section{Haizhen Ma}

Qilu University of Technology (Shandong Academy of Sciences)

\section{Xingwang Liu}

Qilu University of Technology (Shandong Academy of Sciences)

\section{Panpan Li}

Qilu University of Technology (Shandong Academy of Sciences)

\section{Can Li}

Qilu University of Technology (Shandong Academy of Sciences)

\section{Shengkui Zhang}

Qilu University of Technology (Shandong Academy of Sciences)

\section{Xiaohan Wang}

Qilu University of Technology (Shandong Academy of Sciences)

\section{Tao Xia ( $\nabla$ txia@qlu.edu.cn )}

Qilu University of Technology (Shandong Academy of Sciences)

\section{Research Article}

Keywords: $ү$-PGA, maize (Zea mays L.), drought resistance, RNAseq, rhizosphere microbial communities, plant growth promoting bacteria

Posted Date: August 31st, 2021

DOI: https://doi.org/10.21203/rs.3.rs-846088/v1

License: (c) (i) This work is licensed under a Creative Commons Attribution 4.0 International License. Read Full License

Version of Record: A version of this preprint was published at BMC Plant Biology on January 3rd, 2022. See the published version at https://doi.org/10.1186/s12870-021-03392-w. 


\section{Abstract \\ Background}

Compared with other abiotic stresses, drought stress is a serious causal factor leading to crop yield reduction. Poly- $\gamma$-glutamic acid ( $\gamma$-PGA), as an environmentally friendly biomacromolecule, plays an important role in plant growth and regulation.

\section{Results}

In this project, the effect of exogenous application of Y-PGA on drought tolerance of maize (Zea mays. L) and its mechanism were studied. Drought dramatically inhibited the growth and development of maize, but the exogenous application of Y-PGA significantly increased the dry weight of maize and the contents of $A B A$, soluble sugar, proline, chlorophyll and the photosynthetic rate under severe drought stress. RNAseq data showed that $\mathrm{Y}$-PGA may enhance drought resistance of maize by affecting the expression of $A B A$ biosynthesis and signal transduction related genes, photosynthesis-related genes and other stress-responsive genes, which were also confirmed by RT-PCR and promoter motif analysis. In addition, diversity and structure analysis of rhizosphere soil bacterial community demonstrated that $\mathrm{Y}-\mathrm{PGA}$ enriched the plant growth promoting bacteria such as Actinobacteria, Chloroflexi, Firmicutes, Alphaproteobacteria and Deltaproteobacteria. Meanwhile, $\mathrm{Y}$-PGA significantly improved roots development, urease activity and ABA contents of maize rhizospheric soil under drought stress. This study emphasized the possibility of using Y-PGA to improve crop drought resistance and soil environment under drought condition and revealed its preliminary mechanism.

\section{Conclusions}

Exogenous application of poly-y-glutamic acid could significantly enhance the drought resistance of maize by improving photosynthesis, root development and affecting rhizosphere microbial community.

\section{Background}

As one of the major adverse environmental stresses hamper the crop productivity worldwide, drought stress threat is increasing due to the global climate change [1-4]. The growth and development of plants require sufficient water, water shortage can be fatal to crops and leads to the yield losses. Meanwhile, drought stress also cause a series of other problems, such as soil erosion, land desertification, ecosystem destruction and so on [5-7]. The shortage of water resources has been considered to be an urgent global environmental issue. Water scarcity had aroused great concern, and the effect of drought stress on plants is receiving more and more attention. In order to improve the drought resistance of crops, the morphological, physiological, metabolic, molecular and genetic mechanisms of drought resistance of various plants have been systematically studied [7-12]. Conventional breeding, molecular marker- 
assisted selection, plant transgenic technology, exogenous application of hormones (such as ABA) or osmoprotectants (such as glycine betaine and proline) and drip irrigation are all used as the technical strategies to cope with drought stress [13-21].

Poly- $\gamma$-glutamic acid ( $\gamma-P G A)$ is a non-toxic, water-soluble, biodegradable and environment-friendly biopolymer, which is composed of D/L-glutamic acid monomers and fermented by Bacillus subtilis [22, 23]. In line with its different molecular weights, $y$-PGA could be used in many fields, such as food, medicine, cosmetics and agriculture [24]. y-PGA has been paid more and more attention as an environmentally friendly fertilizer synergist because of its strong water solubility and retention, biodegradability and innocuity [25]. Recent studies have found that $y$-PGA plays an important role in plant growth and regulation, and can be used as a water retaining agent and soil conditioner to improve crop productivity [26-28]. It has been reported that exogenous application of $\mathrm{Y}-\mathrm{PGA}$ could significantly enhance the stress resistance of plants [26, 29-31]. Most of the previous studies focused on cold and salt stress of vegetables such as Brassica napus and cucumber. For example, it was found that $\mathrm{Y}$-PGA could increase salt and cold tolerance of Brassica napus by activating the crosstalk between $\mathrm{H}_{2} \mathrm{O}_{2}$ and $\mathrm{Ca}^{2+}$ signals [32] and enhance drought resistance of Brassica napus by promoting ABA accumulation. However, only few studies assessed the effect of $\mathrm{Y}$-PGA on drought resistance of plants, especially crops. And the regulation mechanism of $Y$-PGA on drought resistance of maize remains unclear. Maize is an important crop integrating grain, feed, energy and industrial raw materials, and plays an extremely important role in world food security and economic development [33]. Its yield is always severely affected by drought stress[34]. In this study, the effect of $y$-PGA on the growth of maize seedlings under drought stress was assessed by adding Y-PGA to soil. In addition, RNAseq was performed to study the gene expression of maize leaves after drought stress, and the changes of rhizosphere microbial community after exogenous application of $\mathrm{Y}$-PGA were also studied, so as to understand the mechanism of exogenous application of $\mathrm{Y}-\mathrm{PGA}$ to change drought resistance of maize. So as to understand the mechanism of exogenous application of PGA to change drought resistance of maize.

\section{Results}

\section{Exogenous application of y-PGA enhanced drought resistance of maize}

In order to investigate the effect of exogenous application of $\mathrm{Y}$-PGA on maize under drought stress, the drought-resistant phenotype of maize with different concentrations of $Y$-PGA $(0,50,70,100 \mathrm{mg} / \mathrm{L})$ were examined (Additional file 1: Fig. S1). The results showed that the addition of y-PGA could significantly enhance the drought resistance of maize, even at a lower concentration $(50 \mathrm{mg} / \mathrm{L})$, and could regenerate maize rapidly after rewatering, while most of the control maize showed severe wilting and could not grow again after rewatering. $50 \mathrm{mg} / \mathrm{L} \gamma$-PGA treatment was used for the subsequent experiments. 
Maize treated with $50 \mathrm{mg} / \mathrm{L}$ Y-PGA exhibited a better phenotype after 7 days of drought stress (Fig. 1A). The dry weight, content of ABA, soluble sugar, proline, chlorophyll and the photosynthetic parameters of maize seedlings after 5 days drought treatment were determined. As shown in Fig. 1B, under drought condition, the dry weight of maize treated with $\mathrm{Y}$-PGA $(0.96 \mathrm{~g})$ was significantly higher than that of control maize $(0.39 \mathrm{~g})$, indicating that $\mathrm{Y}-\mathrm{PGA}$ could alleviate the inhibition of drought stress on the growth of maize seedlings. In addition, compared with the control group, the contents of $A B A$, soluble sugar, proline and chlorophyll in $y$-PGA treatment group were $27.46 \%, 43.61 \%, 108 \%$ and $51.51 \%$ higher, respectively (Fig. 1B). This indicated that $\mathrm{Y}$-PGA could promote the accumulation of ABA, soluble sugar, proline and the chlorophyll in maize under drought stress. The photosynthetic parameters of the maize under drought for $5 \mathrm{~d}$ were also measured, the results showed that both the net photosynthetic rate and stomatal conductance of the maize added $\mathrm{Y}$-PGA were significantly higher than the control maize under drought stress (Fig. 1B).

In order to observe the effect of $y-P G A$ on maize growth under drought stress more directly, the simulated drought experiment with $18 \%$ PEG6000 solution was performed. It was found that the fresh weight of leaves and roots in $\mathrm{Y}$-PGA treatment group was higher than that of the control group (Fig. 2), indicating that the drought resistance of leaves and roots in $\mathrm{Y}-\mathrm{PGA}+\mathrm{PEG}$ group was significantly higher than that of the control group.

\section{Y-PGA significantly improved roots development, urease activity and ABA contents of maize rhizospheric soil under drought stress}

It was found that $\mathrm{Y}$-PGA significantly improved the roots development both under the normal condition and drought stress (Fig. 2A). Under normal growing conditions, the maize treated with $y$-PGA had a better developed root system, and the fresh weight of roots was significantly increased than that of the control group. Under PEG simulate drought stress, the roots growth of the control group was significantly inhibited, however, the roots of the maize treated with Y-PGA were little affected by drought stressand the roots fresh weight was significantly higher than that of control group. Since maize rhizospheric soil was closely contacted with the roots, the urease activity (closely related to soil nitrogen transformation) and ABA contents (closely related to the drought resistance) of the maize rhizospheric soil under the severe drought stress were also detected. It was observed that the urease activity of rhizospheric soil of $y-P G A$ treatments was increased by $27.74 \%$, while the ABA contents of $y-P G A$ treatments soil was also increased by $21.70 \%$ (Table 1$)$. 
Table 1

Effect of $y$-PGA on the contents of ABA and Urease activity of maize rhizospheric soil under severe drought stress

\begin{tabular}{|c|c|c|}
\hline Drought & ABA $(\mu g / g D W)$ & $\begin{array}{l}\text { Urease activity } \\
\text { ( } \mu \mathrm{g} \mathrm{NH} 3-\mathrm{N} / \mathrm{g} / 24 \mathrm{~h})\end{array}$ \\
\hline $0 m g / L ~ \gamma-P G A$ & $1.479 \pm 0.011$ & $767.583 \pm 124.714$ \\
\hline $50 \mathrm{mg} / \mathrm{L} \mathrm{Y-PGA}$ & $1.800 \pm 0.002^{* \star}$ & $980.524 \pm 46.475^{\star \star}$ \\
\hline
\end{tabular}

\section{Differentially expressed genes (DEGs) between maize with y-PGA addition and control under drought stress}

In order to explain the mechanism of $\mathrm{Y}^{-P G A}$ in improving the drought resistance of maize, the leaves of $\mathrm{Y}^{-}$ PGA treatment and the control maize under drought condition for 5 days were used for RNA sequencing to identify the DEGs and pathways in response to drought stress. The total raw reads, clean reads, genome mapping ratio, and uniquely mapping ratio were listed in Additional file 10: Table S1. 16126 DEGs were identified and the distribution of the DEGs was illustrated in Fig. 3A. These DEGs were subjected to enrichment analysis of KEGG pathways and Gene Ontology (GO) functions. Based on KEGG pathway analysis, all DEGs were significantly enriched into 6 pathways ( $Q$ value $\leq 0.05$ ), namely photosynthesis-antenna proteins (31 DEGs), photosynthesis (105 DEGs), glyosylate dicarboxylate metabolism (102 DEGs), oxidative phosphorylation (156 DEGs), alanine, aspartate and glutamate metabolism (73 DEGs) and carotenoid biosynthesis pathway (65 DEGs) (Fig. 3B). The results of GO annotation functions enrichment analysis also showed that GO terms such as photosynthesis and photosystem, response to abiotic stimulus, chlorophyll metabolic process, response to biotic stimulus, electron transport chain and so on were significantly enriched (Additional file 2: Fig. S2B). A more detailed classification of the terms of response to abiotic stimulus showed that these DEGs were mainly related to the response to stress (osmotic stress, salt, heat, cold, reactive oxygen species, and hydrogen peroxide), the response to hormone (ABA, JA, and SA), ABA biosynthetic process, chlorophyll metabolic process, proline biosynthetic process, protein folding, and so on (Additional file 2: Fig. S2B).

\section{Y-PGA improved drought resistance of maize by affecting the expression of photosynthesis-related genes}

As known, drought could significantly reduce the photosynthesis capability of plants. However, KEGG analysis showed that under drought stress, compared with the control maize, the photosynthesis related genes of maize treated with y-PGA were significantly enriched (Fig. 3B), with most of related genes were dramatically upregulated. As shown in Fig. 4 and Additional file 10: Table S1, most genes in DEGs of photosystem II complex were upregulated, except PsbA, PsbB, PsbC, PsbE, PsbF and 1 for PsbP, which were downregulated. In photosystem I complex, all of the DEGs were upregulated. In cytochrome $b 6 / f$ complex, 7 genes encoding PetA, 2 genes encoding PetC and 1 genes encoding PetG were upregulated, while only 1 gene encoding PetD and 1 gene encoding PetA were downregulated. In photosynthetic 
electron transport, other 16 genes encoding PetE, PetF, PetH and PetJ were all up-regulated except 3 genes encoding PetF and 2 for PetH,. In F-type ATPase complex, except 1 gene encoding beta, 1 for gamma and $1 \mathrm{~b}$ which were downregulated, the other 14 genes encoding alpha, beta, gamma, delta, epsilon, a, b and c subunits respectively were upregulated. Additionally, all DEGs (67 genes) encoding antenna proteins were also up-regulated (Additional file 3: Fig. S3). To confirm the results, 14 genes with different transcript abundances were validated by real-time RT-PCR (Additional file 4: Fig. S4). The expression of these genes showed good consistency between the two detection methods. Meanwhile, the motifs in the promoter region of these genes were analyzed, higher percentage of drought, lowtemperature, salicylic stress and ABA response elements were found (Additional file 5: Fig. S5, Additional file 6: Fig. S6).

\section{Y-PGA promoted $A B A$ accumulation and affected $A B A$ signaling to improve drought resistance of maize}

$A B A$, as an important drought response hormone, plays an important role in the response of maize to abiotic stress. Based on KEGG pathway analysis, it was found that DEGs related with carotenoid biosynthesis pathway which contains ABA biosynthesis pathway were significantly enriched (Fig. 3B), $\mathrm{Y}^{-}$ PGA could promote ABA accumulation under drought condition (Fig. 1B). CHY2, ABA1, NCED, ABA2 and $A A O 3$ were reported to be involved in $A B A$ biosynthesis [35-38], 8'-hydroxyase was reported to play an important role in the catabolism of ABA [39]. RNAseq results showed that 2 genes encoding $\mathrm{CHY} 2,7$ genes encoding $A B A 1,3$ genes encoding NCED, 2 genes encoding $A B A 2$, and 1 genes encoding AAO3 were significantly upregulated, while 2 genes encoding 8'-hydroxyase were downregulated. In addition, $A B A$ signaling pathway related genes, including ABA receptor (PYR/PYL), PP2C, SnRK2 and ABFs were also differentially expressed. Among these DEGs, 3 for PYL, 4 for SnRK2, and 4 for ABF were upregulated, 10 for PP2C were downregulated (Additional file 7: Fig. S7).

\section{Y-PGA affected the bacterial community diversity and structure of rhizospheric soil}

In order to study the influence of $\mathrm{Y}$-PGA on bacterial community diversity under drought stress, the relative abundance and diversity of maize rhizospheric soil bacteria were analyzed by high-throughput sequencing of $16 \mathrm{~S}$ rRNA. The species curve showed that the samples were representative enough to obtain a true bacterial community (Additional file 8: Fig. S8). NMDS (stress $=0.00422$ ) of the weighted UniFrac distance ordinations were conducted (Fig. 5A), the results indicated that the bacterial community composition of the soil with $y$-PGA application brought shifts compared with that of the soil without $\mathrm{Y}^{-}$ PGA under the drought stress, the communities in maize rhizospheric soil with $y$-PGA were grouped together and significantly separated from those in soil without $\gamma$-PGA under the drought stress. The obtained high-quality sequences were belonged to 36 phylum,among which the main phylum was Proteobacteria, followed by Actinobacteria, Chloroflexi, Bacteroidetes, and Acidobacteria. Although the diversity of bacterial community changed after the addition of $y$-PGA under drought stress $\square$ the predominant phylum were similar. There was no difference in species composition among these samples, but the relative abundances of some species changed (Fig. 5B). Compared to the control, the relative abundance of Actinobacteria and Chloroflexi were higher in soil added $\mathrm{Y}$-PGA under drought stress. LEfSe 
analysis ( $L D A \geq 3$ ) showed the species with the most significant variation (Fig. 5C). Under drought stress, the application of $\mathrm{Y}$-PGA could significantly enrich Actinobacteria, Chloroflexi and Cyanobacteria at phylum level, while Alphaproteobacteria and Deltaproteobacteria were enriched at class level. At the genus level, bacteria such as Rhodobacter Sphingobium, Sphingomonas, Sphingopyxis, Haliangium, Methylibium, Lysobacter, Azoarcus and Arenimonas of Proteobacteria, Aeromicrobium, Lechevalieria and Streptomyces of Actinobacteria, Subgroup_10 of Acidobacteria, Clostridium and Pelotomaculum of Firmicutes, Chloronema, A4b and KD4-96 of Chloroflexi were dominant in Y-PGA added rhizosphere soil under the persistent severe drought condition. The abundances of these genera in maize rhizospheric soil with $\mathrm{Y}$-PGA addition were all higher than that of control (Additional file 9: Fig. S9), while Bacillus of Proteobacteria was dominated in control (Fig. 5C).

\section{Discussion}

Among all abiotic stresses, drought has the greatest impact on soil organisms and plants [40]. Drought could adversely affect the important physiological and biochemical processes of plants, resulting in serious loss of crop yield worldwide [41]. It is critical to improve the plant tolerance to drought stress. As a natural and environment friendly biopolymer, $\mathrm{Y}$-PGA has been widely used in agricultural production [42]. However, there are few reports about the effect and mechanism of $Y$-PGA on drought resistance of plants, especially crops. In this study, the effect of $y-P G A$ on maize drought resistance and its comprehensive mechanism by RNAseq and rhizosphere soil bacterial community diversity analysis were firstly reported.

The effects of exogenous application of $\mathrm{Y}-\mathrm{PGA}$ on dry weight, the contents of $A B A$, soluble sugar, proline and chlorophyll of maize leaf under severe drought stress were characterized. These physiological indexes have been often used to evaluate the drought resistance of plants. As the osmoprotectants, proline and soluble sugar could provide osmotic adjustments in plants under drought stress[43]. Proline has strong hydration ability, which can protect cell structure and enzymes, reduce cell acidity and regulate redox potential under stress. $A B A$ is considered to be the most critical hormone regulating tolerance to drought stress. Drought stress could trigger a huge increase in ABA biosynthesis. As a key chemical messenger of drought signal, $A B A$ could activate a series of signal transduction reactions to regulate stomatal closure, calcium signal and the expression of some ABA-responsive genes to resist the drought stress. Drought stress can significantly decline the chlorophyll content of leaves [44, 45]. Plants with higher chlorophyll contents under drought stress could use light energy more efficiently and have better drought resistance. In this study, we found that under drought stress, $y$-PGA could promote the accumulation of $A B A$, soluble sugar, proline and chlorophyll, the drought resistance of maize was significantly enhanced by adding $Y$-PGA. In addition, $\gamma$-PGA could increase the dry weight of maize under drought stress, indicating that maize added with $Y$-PGA could still maintain a certain growth compared with that of control. In order to observe the root morphology under drought stress more directly, PEG6000 was used to simulate the drought treatment in the solution culture process. The results showed that, under PEG treatment, maize added with y-PGA had more developed roots than that of control, which could make maize absorb deeper and more water of soil during drought stress. 
To explore the molecular mechanism of enhanced drought resistance by exogenous application of $\mathrm{Y}-\mathrm{PGA}$, the differentially expressed genes (DEGs) of the leaves were evaluated by RNAseq analysis. KEGG analysis showed that photosynthesis related genes were significantly enriched which was consistent with the increase of photosynthetic rate in the maize treatment with $y$-PGA under the drought stress. Most of the photosynthesis related genes, including 20 genes in photosystem I, 28 genes involved in photosystem II, 16 genes in photosynthetic electron transport, 10 genes in cytochrome b6/f complex, 14 genes in ATPase complex, and 31 genes encoding antenna proteins (9 genes encoding $\mathrm{LHCl}$ complex, 22 genes encoding LHCII complex), were dramatically upregulated in Y-PGA treatment maize compared with that of control. Photosynthesis is one of the main processes affected by drought [46]. However, under severe drought stress, the photosynthesis related genes in maize added with Y-PGA still maintained a high expression level than that of control, which may be the main reason for the higher drought resistance of maize treated with y-PGA, while the reduced chlorophyll contents under drought in control leaded to the inactivation of photosynthesis.

$A B A$ is considered to be the most critical hormone involved in the adaptive responses of plants to drought stress. DEGs related with carotenoid biosynthesis pathway which contains ABA biosynthesis pathway were also found to be significantly enriched in this study. In ABA biosynthesis, $\beta$-carotene is converted to zeaxanthin by $\mathrm{CHY} 2$ enzyme firstly, the epoxidation of zeaxanthin and antheraxanthin to violaxanthin was catalyzed by zeaxanthin epoxidase (ZEP/ABA1) afterwards [35]. Violaxanthin is converted to 9-cisviolaxanthin after a series of structural modifications. The next step is also a rate-limiting step, that is, 9cis-violaxanthin is converted to xanthoxin under the catalysis of 9-cis-epoxycarotenoid dioxygenase (NCED) [36]. Subsequently, xanthoxin is converted to abscisic aldehyde, and then ABA is produced by two-step reaction via ABA-aldehyde. The enzyme (alcohol dehydrogenase/reductase) encoded by ABA2 catalyzes the first step of this reaction and generates ABA aldehyde[37], and abscisic aldehyde oxidase encoded by AAO3 catalyzes the last step of ABA synthesis [38]. In this study, it was found that the ABA biosynthesis related genes including 2 genes encoding $C H Y 2,7$ genes encoding $A B A 1,3$ genes encoding NCED, 2 genes encoding $A B A 2$, and 1 genes encoding $A A O 3$ were significantly upregulated, while 2 genes encoding 8'-hydroxyase which played important role in the catabolism of ABA were downregulated in maize with the application of $y$-PGA. The expression level of these DEGs led to the increase of ABA level in $\gamma$-PGA treated maize under drought stress. The results indicated that $\gamma$-PGA could promote ABA accumulation under drought condition, and the accumulation of $A B A$ can activate the core ABA signaling pathway including PYR/PYL/RCAR receptor, PP2C proteins, SnRK2 family members, AREB/ABF transcription factors and downstream regulatory genes, as well as ABA-activated signaling pathway to resist drought stress [47]. In addition, many reports have shown that among the promoters of the stressresponsive genes, there was a major cis-acting element (ABRE) which was regarded to be necessary for $A B A$ response [48]. We found that ABRE element were present in the promoters of these upregulated photosynthesis related genes, suggesting that these genes may also be regulated by ABA. In addition, it was also found that many stress-responsive genes, including the DEGs response to abiotic stimulus, were significantly enriched (Fig. 6). 
Many reports have shown that drought stress has a great impact on soil microbial communitieswhich play an important role in regulating plant response to drought stress [49]. Drought stress could lead to a significant reduction of microbial biomass [50-52] and change the composition of plant rhizosphere microbial. The drought tolerance of plants is related to the change of relative abundance of specific bacterial groups $[29-32,40]$. Although our understanding of the interaction between plants and soil microbial in drought responses is advancing, most of the knowledge comes from non-crop plants. The results in this study showed that the application of $\mathrm{Y}$-PGA under the drought stress did not affect the species of dominant bacteria, but change the bacterial community diversity. Under drought stress, Actinobacteria and Chloroflexi were significantly enriched in soil supplemented with y-PGA (Fig. 5C). Actinobacteria and Chloroflexi were reported to be the most prominent phylum of drought enrichment [53]. Actinobacteria was previously found to promote the decomposition or formation of humus, making it easier to be absorbed $[54,55]$, and it was also reported to have the important role in plant defense and growth promotion [56-58]. In this study, it was also found that Alphaproteobacteria and Deltaproteobacteria were enriched at class level after addition of $\mathrm{Y}$-PGA. Most members of Proteobacteria were reported to play important roles in nitrogen fixation $[59,60]$. Exogenous application of $Y$-PGA under drought stress could also enrich Sphingobium, Sphingomonas, Sphingopyxis, Haliangium of Proteobacteria. In addition, Subgroup_10 of Acidobacteria, Clostridium and Pelotomaculum of Firmicutes, which was previously reported to promote plant growth through nitrogen fixation, phosphate solubilization and production of plant hormone [54], were also found to be significantly enriched in the $\gamma^{-}$ PGA added soil in this study.

It is worth noting that $y-P G A$ increased the urease activity of rhizosphere soils of maize under the severe drought stress (Table 2). The activities of soil urease play an important role in soil nitrogen transformation, which produces $\mathrm{NH}_{3}, \mathrm{NH}_{4}{ }^{+}$and $\mathrm{CO}_{3}{ }^{2-}$ in the process of urea hydrolysis and provides nutrition for plants. The results implied that exogenous application of $\mathrm{y}$-PGA could contribute to improve the soil biochemical reaction and plant growth under the drought stress condition. In addition, interestingly, we also detected a significant increase in ABA content in the rhizosphere soil after y-PGA application, which will also play an important role in drought resistance of maize. The mechanism of the increase of urease activity and ABA content in soil by exogenous application of $\mathrm{Y}$-PGA needs further study. Our results showed that exogenous application of PGA not only affected the physiological and biochemical indexes and gene expression related to drought resistance of plants, but also profoundly affected the microbial community and physiological and biochemical properties of rhizosphere soil.

\section{Conclusions}

Our study demonstrated that exogenous application of $y-P G A$ could significantly enhance the drought resistance of maize under severe drought stress. $y$-PGA can regulate the expression of ABA biosynthesis, ABA signal transduction related genes, photosynthesis-related genes and other stress-responsive genes. At the same time, $\mathrm{y}$-PGA could enrich the plant-promoting bacteria such as Actinobacteria, Chloroflexi, 
Firmicutes, Alphaproteobacteria and Deltaproteobacteria. This study highlighted the possibility of using Y- $^{-}$ PGA to improve crop drought resistance and soil environment under drought condition.

\section{Materials And Methods}

\section{Plant materials and drought treatments}

Maize (inbred line KN5585) seeds (provied by Weimi Biotechnology (Jiangsu) Co., Ltd (Changzhou, China)) were sown in a soil box $(10 \mathrm{~cm} * 10 \mathrm{~cm} * 10 \mathrm{~cm})$. When seeds germinated, the seedlings were watered with different concentrations $(0,50,70,100 \mathrm{mg} / \mathrm{L})$ of $\mathrm{Y}$-PGA (10KD) solution区and grown in greenhouse at $28 \pm 2^{\circ} \mathrm{C}$ under nature light $\$ and $25 \pm 2^{\circ} \mathrm{C}$ at night. All seedlings at three-leaf stage were exposed to a drought stress treatment by stopping watering to select the most suitable treatment concentration of $\mathrm{Y}$-PGA. After drought for 7 days (the soil water content decreased to $4.9 \%$, and the control plants wilted seriously), the seedlings were rewatered. After rewatering for 1 day, the recovered maize added with $\gamma$-PGA were recorded and compared with the control maize. $50 \mathrm{mg} / \mathrm{L} \gamma$-PGA was selected for the subsequent experiment according to the results of drought lethal test. The physiological parameters including photosynthetic parameters (net photosynthetic $\mathrm{CO}_{2}$ assimilation rate, stomatal conductance), soluble sugar, proline, chlorophyll and ABA contents of the maize added with $y$-PGA and the control maize were measured after 5 days treatment (soil water content decreased to $9.8 \%$ ). Soil water content was monitored by using Soil Moisture Content Meter (TZS, TOP instrument, China). Each experiment had at least three biological repetitions, and the determination of photosynthetic parameters was repeated at least five times. The leaves were taken for RNA sequencing. Finally, the dry weights of plants under drought conditions were measured.

In the experiment of using PEG to simulate drought stress, maize (inbred line KN5585) seeds were surface sterilized using $75 \%$ alcohol and germinated on moist filter paper in sterile petri dishes (diameter: $12.5 \mathrm{~cm}$ ) in the dark at $28^{\circ} \mathrm{C}$. After 4 days, the germinated seeds were transferred to the culture flasks (height: $15 \mathrm{~cm}$, diameter: $7 \mathrm{~cm}$ ) with Hoagland Solution, and grown at $28^{\circ} \mathrm{C} / 25^{\circ} \mathrm{C}(16 \mathrm{~h} \mathrm{light} / 8 \mathrm{~h}$ dark) until maize reached to two-leaf stage. Then the maize seedlings were divided into four groups and cultured as follows: group1, cultured with Hoagland Solution only; group2, cultured with Hoagland Solution supplemented with $18 \%(\mathrm{~m} / \mathrm{v})$ PEG6000 (-0.77 MPa) solution; group3, cultured with Hoagland Solution supplemented with Y-PGA $\left(10 \mathrm{kDa}, 50 \mathrm{mg} \mathrm{L}^{-1}\right)$; group 4, cultured with Hoagland Solution supplemented with $\mathrm{Y}$-PGA $\left(10 \mathrm{kDa}, 50 \mathrm{mg} \mathrm{L}^{-1}\right)+18 \%(\mathrm{~m} / \mathrm{v})$ PEG6000. The nutrient solution was renewed every 2 days, aerated with a mini air pump and supplemented with fresh solution. The phenotypes of plants were examined, the leaf and root fresh weight were measured.

\section{Determination of physiological parameters}

The leaves and roots disk from the plants were excised, and the fresh weights (FWs) were recorded immediately. The dry weights (DWs) of leaves were obtained after drying in an oven at $80^{\circ} \mathrm{C}$. The photosynthetic parameters (net photosynthetic $\mathrm{CO}_{2}$ assimilation rate and stomatal conductance) were 


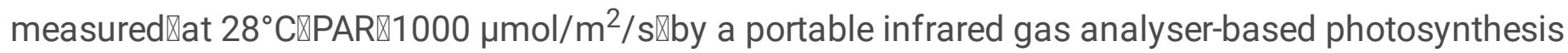
system (Yaxin-1105, China). Total soluble sugars of leaves (approximately $100 \mathrm{mg}$ ) were extracted in boiling water for $30 \mathrm{~min}$ and determined by anthrone reagent using glucose as the standard according to the methods described by Yemm and Willis [61]. Proline was detected using the protocol described by Bates et al [62]. Approximately $200 \mathrm{mg}$ of the maize leaves was excised to measure chlorophyll content following the method described by Arnon [63]. The ABA content was measured with ELISA kit (code JM01148P2, Jingmei Bio Inc,. Jiangsu, China) according to the manufacturer's protocol. The urease activity was determined according to the described method by Guan [64]. In this study, at least three biological repeats were sampled for one treatment, each replicate contained tissues from four plants, and the determination of photosynthetic parameters was repeated at least five times.

\section{RNA extraction and real-time RT-PCR}

Total RNA was isolated from maize leaves as described by the manufacturer's instructions using HiPure RNA Kit (Magen, Guangzhou, China). $2 \mu \mathrm{g}$ of total RNA was reverse transcribed into CDNA using Reverse transcription kit (TAKARA). The cDNA was diluted to $200 \mu \mathrm{L}$ by sterile DEPC water. Real-time RT-PCR of the candidate genes were performed by SYBR Green I Master Mix (Roche, Indianapolis, USA). Three biological and three technical replicates for each reaction were analyzed on LightCycler 480 (Roche, USA) with the first step of $95^{\circ} \mathrm{C}$ for $5 \mathrm{~min}$ followed by 40 cycles of $95^{\circ} \mathrm{C}$ for $10 \mathrm{~s}, 60^{\circ} \mathrm{C}$ for $10 \mathrm{~s}$, and $72{ }^{\circ} \mathrm{C}$ for $20 \mathrm{~s}$. Melting curves were generated using the following program: $95^{\circ} \mathrm{C}$ for $15 \mathrm{~s}, 60^{\circ} \mathrm{C}$ for $15 \mathrm{~s}$, and for $15 \mathrm{~s}$. $Z m T u b$ was used as an internal control. Data analysis was calculated by $2^{-\Delta \Delta C T}$ method. Significant differences between different samples were tested with IBM SPSS Statistics 22.0 software. Real-time PCR of the candidate genes and data analysis was performed and primers used were list in Additional file 11: Table S2.

\section{RNA sequencing and analysis}

RNA sequencing and primary bioinformatics analysis were performed by BGI Tech Solutions Co., Ltd. (She nzhen, China). Each treatment was made three biological replicates. Primary sequencing data (raw read) were produced by Illumina HiSeq ${ }^{\mathrm{TM}} 2000$. After QC, raw reads were filtered into clean reads which will be aligned to the reference sequences. The alignment data was utilized to calculate distribution of reads on reference genes and mapping ratio. Gene expression was measured as fragments per kilobase of transcript per million fragments mapped (FPKM) using Cufflinks. Differentially expressed genes (DEGs) were determined using DEseq2. The false discovery rate was used to adjust the P-values. Genes with significant differences in expression, llog2Fold Changel $\geq 1$, and adjusted $P$-value $<0.05$ were considered as DEGs. GO analysis and pathway enrichment analysis of all DEGs ( $Q$ value $\leq 0.05$ ) were performed by AgriGO (http:// bioinfo.cau.edu.cn/agriGO/) and KEGG (http://www.genome.jp/kegg/). The promoter motif analysis was conducted using PlantCARE (http://bioinformatics.psb.ugent.be/webtools/plantcare/html/).

\section{Bacterial Community Analysis of maize rhizosphere soil}


The two-leaf stage maize seedlings watered with $y-P G A(0,50 \mathrm{mg} / \mathrm{L})$ were treated under drought stress and kept the soil moisture content at $8.0 \%$ by replenishment. After 30 days, the tightly bound soils of roots (served as rhizosphere soils) were taken to analyze the microbial community, and three biological replicates were performed. Amplification and High-throughput sequencing of 16s rRNA from maize rhizosphere soil bacterial were performed as described by Wang et al.[65]. The primers of V4 region of bacterial 16S rRNA were 338F (5'-ACTCCTACGGGAGGCAGCA-3') and 806R (5'-

GGACTACHVGGGTWTCTAAT-3'). High-throughput sequencing was conducted by Illumina Hiseq 2000 (Illumina Inc., San Diego, USA). Nonmetric multidimensional scaling (NMDS) was performed on distance matrices and the coordinates were used to draw 2D graphical outputs. Taxa abundances at the phylum, class, order, family and genus levels were statistically compared among samples or groups by Metastats. The LEfSe analysis (LDA $\geq 3$ ) was carried out to obtain the important indicator taxa with significant changes in relative abundance.

\section{Statistical Analysis}

All data have at least three biological replicates. The data were presented as the mean \pm standard deviation (SD). The statistical analysis between the maize with and without $y$-PGA was performed using T-test and Duncan's tests of one-way ANOVAs in SPSS (version 22.0.0.0). Significant differences were indicated by asterisks, ${ }^{\star} \mathrm{p}<0.05 ;{ }^{\star \star} \mathrm{p}<0.01$.

\section{Declarations}

\section{Ethics approval and consent to participate}

There is no ethics approval and consent to participate in this manuscript.

\section{Consent to publication}

Not applicable.

\section{Competing interests}

The authors declare no conflict of interest.

\section{Availability of data and materials}

All datasets generated for this study are included in the article/Supplementary Materials.

The RNA-Seq raw data have been uploaded to a public database: https://doi.org/10.6084/m9.figshare.14495775.v1

The data of 16s rRNA from maize rhizosphere soil bacterial were deposited in the figshare database: https://doi.org/10.6084/m9.figshare.14496006.v1 


\section{Funding}

This study was financially supported by the Integration of Science and Education Program Foundation for the Talents by Qilu University of Technology, Shandong Academy of Sciences (No. 2018-81110268), Foundation of State Key Laboratory of Biobased Material and Green Papermaking (No. 2419010205, No. 23190444 and No. ZZ20200130).

\section{Authors' contributions}

Tao Xia and Haizhen Ma designed the research project. Haizhen Ma performed the experiments and analyzed the data. Xingwang Liu凶Panpan Li, Can Li, Shengkui Zhang and Xiaohan Wang assisted in the determination of some physiological indexes, drought stress treatment and bacterial community analysis. Haizhen Ma and Tao Xia wrote this manuscript.

\section{Acknowledgments}

We would like to thank to BGI Tech Solutions Co., Ltd (Shenzhen, China) for Illumina sequencing and primary bioinformatics analysis.

\section{References}

1. Kakumanu A, Ambavaram MM, Klumas C, Krishnan A, Batlang U, Myers E, Grene R, Pereira A: Effects of drought on gene expression in maize reproductive and leaf meristem tissue revealed by RNA-SEq. Plant physiology 2012, 160(2):846-867.

2. Shah F, Bajwa AA, Usman N, Anjum SA, Ayesha F, Ali Z, Sehrish S, Wajid N, Steve A, Shah S: Crop Production under Drought and Heat Stress: Plant Responses and Management Options. Frontiers in plant science 2017, 8:1147-.

3. Yamaguchi-Shinozaki K, Shinozaki K: TRANSCRIPTIONAL REGULATORY NETWORKS IN CELLULAR RESPONSES AND TOLERANCE TO DEHYDRATION AND COLD STRESSES. Annual review of plant biology 2006, 57(1):781-803.

4. Bray EA: Plant responses to water deficit. Trends in plant science 1997, 2(97):48-54.

5. Wada Y, Beek LPHV, Bierkens MFP: Modelling global water stress of the recent past: on the relative importance of trends in water demand and climate variability. Hydrology \& Earth System Sciences 2011, 8(12):3785-3805.

6. Gerard, van, der, Schrier, Jonathan, Barichivich, Aiguo, Da, Justin, Sheffield: Global warming and changes in drought. Nature Climate Change 2014, 4(1):17-22.

7. Fang Y, Xiong L: General mechanisms of drought response and their application in drought resistance improvement in plants. Cellular \& Molecular Life Sciences Cm/s 2015, 72(4):673.

8. Chaves, Flexas, Pinheiro: Photosynthesis under drought and salt stress - regulation mechanisms from the whole plant to cell. PHOTOSYNTH RES 2007. 
9. Nezhadahmadi A, Prodhan ZH, Faruq G: Drought tolerance in wheat. TheScientificWorldJournal 2013, 2013:610721.

10. Martignago D, Rico-Medina A, Blasco-Escámez D, Fontanet-Manzaneque JB, Cao-Delgado Al: Drought Resistance by Engineering Plant Tissue-Specific Responses. Frontiers in plant science 2020, 10.

11. Goswami, A., Banerjee, R., Raha, S.: Drought resistance in rice seedlings conferred by seed priming: Role of the anti-oxidant defense mechanisms. Protoplasma: An International Journal of Cell Biology 2013, 250(5):1115-1129.

12. Fàbregas N, Fernie AR: The metabolic response to drought. Journal of Experimental Botany 2019, 70(4):1077-1085.

13. Hu H, Xiong L: Genetic engineering and breeding of drought-resistant crops. Annual Review of Plant Biology 2014, 65(1):715-741.

14. Fukai S, Cooper M: Development of drought-resistant cultivars using physiomorphological traits in rice. Field Crops Res 1995, 40(2):67-86.

15. Wu Y, Chen $\mathrm{H}$, Chen F, Chu C: Overexpression of a rice OsDREB1F gene increases salt, drought, and low temperature tolerance in both Arabidopsis and rice. Plant Molecular Biology 2008, 67(6):589602.

16. Mark, Tester, Peter, Langridge: Breeding Technologies to Increase Crop Production in a Changing World. Science (New York, NY) 2010.

17. Passioura JB, Spielmeyer W, Bonnett DG: Requirements for Success in Marker-Assisted Breeding for Drought-Prone Environments: Springer Netherlands; 2007.

18. Dixit S, Singh A, Sandhu N, Bhandari A, Vikram P, Kumar A: Combining drought and submergence tolerance in rice: marker-assisted breeding and QTL combination effects. Mol Breed 2017, 37(12):143.

19. Jianhua, Zhang, and, Wensuo, Jia, and, Jianchang, Yang, and, Abdelbagi: Role of ABA in integrating plant responses to drought and salt stresses. Field Crops Research 2006.

20. Per TS, Khan NA, Reddy PS, Masood A, Hasanuzzaman M, Khan MIR, Anjum NA: Approaches in modulating proline metabolism in plants for salt and drought stress tolerance: Phytohormones, mineral nutrients and transgenics. Russian Journal of Plant Physiology 2017, 115:126-140.

21. Ashraf M, Foolad MR: Roles of glycine betaine and proline in improving plant abiotic stress resistance. Environmental and experimental botany 2007.

22. Ashiuchi M, Kamei T, Baek DH, Shin SY, Sung MH, Soda K, Yagi T, Misono H: Isolation of Bacillus subtilis (chungkookjang), a poly-gamma-glutamate producer with high genetic competence. Applied Microbiology \& Biotechnology 2001, 57(5-6):764-769.

23. Luo Z, Guo Y, Liu J, Qiu H, Zhao M, Zou W, Li S: Microbial synthesis of poly-y-glutamic acid: current progress, challenges, and future perspectives. Biotechnology for Biofuels 2016, 9(1):134. 
24. Shih IL, Van YT: The production of poly-(gamma-glutamic acid) from microorganisms and its various applications. Bioresour Technol 2001, 79(3):207-225.

25. Ogunleye A, Bhat A, Irorere VU, Hill D, Williams C, Radecka I: Poly-Y-glutamic acid: production, properties and applications. Microbiology 2014.

26. Guo Z, Yang N, Zhu C, Gan L: Exogenously applied poly-y-glutamic acid alleviates salt stress in wheat seedlings by modulating ion balance and the antioxidant system. Environmental science and pollution research international 2017, 24(7):6592-6598.

27. Xu Z, Lei P, Feng X, Xu X, Tang W: Effect of poly(Y -glutamic acid) on microbial community and nitrogen pools of soil. Acta Agriculturae Scandinavica, Section B - Soil \& Plant Science 2013, 63(8):657-668.

28. Xu Z, Wan C, Xu X, Feng X, Xu H: Effect of poly (Y-glutamic acid) on wheat productivity, nitrogen use efficiency and soil microbes. Journal of Soil Science \& Plant Nutrition 2013, 13(3):744-755.

29. Xu Z, Lei P, Pang X, Li H, Feng X, Xu H: Exogenous application of poly-glutamic acid enhances stress defense in Brassica napus $\mathrm{L}$. seedlings by inducing cross-talks between $\mathrm{Ca} 2+, \mathrm{H} 2 \mathrm{O} 2$, brassinolide, and jasmonic acid in leaves. Plant Physiology \& Biochemistry 2017, 118:460-470.

30. Lei P, Xu Z, Ding Y, Tang B, Zhang Y, Li H, Feng X, Xu H: Effect of Poly(Y-glutamic acid) on the Physiological Responses and Calcium Signaling of Rape Seedlings (Brassica napus L.) under Cold Stress. Journal of agricultural and food chemistry 2015, 63(48):10399-10406.

31. Lei P, Xu Z, Liang J, Luo X, Xu H: Poly(Y-glutamic acid) enhanced tolerance to salt stress by promoting proline accumulation in Brassica napus L. Plant Growth Regulation 2016, 78(2).

32. Effect of $\mathrm{Y}$-PGA coated urea on N-release rate and tomato growth. Wuhan University Journal of Natural Sciences 2014, 19(4):335-340.

33. Gong F, Yang L, Tai F, Hu X, Wang W: "Omics" of Maize Stress Response for Sustainable Food Production: Opportunities and Challenges. Omics A Journal of Integrative Biology 2014, 18(12):714732.

34. Wang B, Liu C, Zhang D, He C, Li Z: Effects of maize organ-specific drought stress response on yields from transcriptome analysis. BMC Plant Biology 2019, 19(1).

35. Thompson AJ, Jackson AC, Parker RA, Morpeth DR, Burbidge A, Taylor IB: Abscisic acid biosynthesis in tomato: regulation of zeaxanthin epoxidase and 9-cis-epoxycarotenoid dioxygenase mRNAs by light/dark cycles, water stress and abscisic acid. Plant Molecular Biology 2000, 42(6):833-845.

36. Tan BC, Schwartz SH, Zeevaart JAD, Mccarty DR: Genetic control of abscisic acid biosynthesis in maize. Proc Nat Acad Sci USA 94: 12235-12240. Proceedings of the National Academy of Sciences 1997, 94(22):12235-12240.

37. González-Guzmán M, Apostolova N, Bellés JM, Barrero JM, Rodríguez PL: The short-chain alcohol dehydrogenase ABA2 catalyzes the conversion of xanthoxin to abscisic aldehyde. Plant Cel/ 2002, 14(8):1833.

38. Seo M, Peeters AJM, Koiwai H, Oritani T, Koshiba T: The Arabidopsis aldehyde oxidase 3 (AA03) gene product catalyzes the final step in abscisic acid biosynthesis in leaves. Proceedings of the 
National Academy of Sciences 2000, 97(23):12908-12913.

39. Chono M, Matsunaka H, Seki M, Fujita M, Kiribuchi-Otobe C, Oda S, Kojima H, Kobayashi D, Kawakami N: Isolation of a wheat (Triticum aestivum L.) mutant in ABA 8'-hydroxylase gene: effect of reduced ABA catabolism on germination inhibition under field condition. Breeding Science 2013, 63(1):104-115.

40. Zia R, Nawaz MS, Siddique MJ, Hakim S, Imran A: Plant survival under drought stress: Implications, adaptive responses, and integrated rhizosphere management strategy for stress mitigation. Microbiological research 2021, 242:126626.

41. Bodner G, Nakhforoosh A, Kaul HP: Management of crop water under drought: a review. Agronomy for Sustainable Development 2015, 35(2):401-442.

42. Xu Z, Ma J, Lei P, Wang Q, Xu H: Poly-Y-glutamic acid induces system tolerance to drought stress by promoting abscisic acid accumulation in Brassica napus L. Scientific reports 2020, 10(1):252.

43. Per TS, Khan NA, Reddy PS, Masood A, Hasanuzzaman M, Khan MIR, Anjum NA: Approaches in modulating proline metabolism in plants for salt and drought stress tolerance: Phytohormones, mineral nutrients and transgenics. Plant Physiology and Biochemistry 2017.

44. Rong-Hua LI, Pei-Guo G, Baum M, Grando S, Ceccarelli S: Evaluation of Chlorophyll Content and Fluorescence Parameters as Indicators of Drought Tolerance in Barley. Agricultural Sciences in China 2006, 5(10):751-757.

45. Kocheva K, Lambrev P, Georgiev G, Goltsev V, Karabaliev M: Evaluation of chlorophyll fluorescence and membrane injury in the leaves of barley cultivars under osmotic stress. Bioelectrochemistry 2004, 63(1-2):121-124.

46. Chaves MM, Flexas J, Pinheiro C: Photosynthesis under drought and salt stress: regulation mechanisms from whole plant to cell. Annals of botany 2009, 103(4):551-560.

47. Cutler SR, Rodriguez PL, Finkelstein RR, Abrams SR: Abscisic Acid: Emergence of a Core Signaling Network. Annual Review of Plant Biology 2010, 61(1):651-679.

48. Hattori T: Experimentally Determined Sequence Requirement of ACGT-Containing Abscisic Acid Response Element. Plant and Cell Physiology 2002, 43(1):136-140.

49. Leng G, Hall J: Crop yield sensitivity of global major agricultural countries to droughts and the projected changes in the future. The Science of the total environment 2019, 654:811-821.

50. Naylor D, Coleman-Derr D: Drought Stress and Root-Associated Bacterial Communities. Frontiers in plant science $2017,8: 2223$.

51. Jansson JK, Hofmockel KS: Soil microbiomes and climate change. 2020, 18(1):35-46.

52. de Vries FT, Griffiths RI, Knight CG, Nicolitch O, Williams A: Harnessing rhizosphere microbiomes for drought-resilient crop production. Science (New York, NY) 2020, 368(6488):270-274.

53. Santos-Medellín C, Edwards J, Liechty Z, Nguyen B, Sundaresan V: Drought Stress Results in a Compartment-Specific Restructuring of the Rice Root-Associated Microbiomes. mBio 2017, 8(4):e00764-00717. 
54. Yin A, Jia Y, Qiu T, Gao M, Cheng S, Wang X, Sun Y: Poly-Y-glutamic acid improves the drought resistance of maize seedlings by adjusting the soil moisture and microbial community structure. Applied Soil Ecology 2018:S0929139318302324.

55. M.Buée, Reich M, Murat C, Morin E, Nilsson RH, Uroz S, Martin F: 454 Pyrosequencing analyses of forest soils reveal an unexpectedly high fungal diversity. New Phytologist 2010, 184(2):449-456.

56. Franco CM, Conn V, Walker A: Endophytic Actinobacteria induce defense pathways in Arabidopsis Thaliana. Molecular Plant Microbe Interactions 2008, 21(2):208-218.

57. Cha JY, Han S, Hong HJ, Cho H, Kwak YS: Microbial and biochemical basis of a Fusarium wiltsuppressive soil. Isme Journa/ 2016, 10(1):119-129.

58. Palaniyandi SA, Yang SH, Zhang L, Suh JW: Effects of actinobacteria on plant disease suppression and growth promotion. Applied Microbiology \& Biotechnology 2013, 97(22):9621-9636.

59. García-Salamanca A, Molina-Henares MA, Dillewijn PV, Solano J, Pizarro-Tobías P, Roca A, Duque E, Ramos JL: Bacterial diversity in the rhizosphere of maize and the surrounding carbonate-rich bulk soil. Microbial Biotechnology 2013.

60. Johnston-Monje D, Lundberg DS, Lazarovits G, Reis VM, Raizada MN: Bacterial populations in juvenile maize rhizospheres originate from both seed and soil. Plant \& Soil 2016, 405(1):337-355.

61. Yemm EW, Willis AJ: The estimation of carbohydrates in plant extracts by anthrone. Biochem $\mathrm{J} 1954$, 57(3):508-514.

62. Bates LS, Waldren RP, Teare ID: Rapid Determination of Free Proline for Water-Stress Studies. Plant and Soil 1973, 39(1):205-207.

63. Arnon DI: COPPER ENZYMES IN ISOLATED CHLOROPLASTS. POLYPHENOLOXIDASE IN BETA VULGARIS. Plant Physiology 1949(1):1.

64. Guan SY, Zhang D, Zhang Z: Soil enzyme and its research methods. 1986.

65. Wang X, Dong G, Liu X, Zhang S, Li C, Lu X, Xia T: Poly-Y-glutamic acid-producing bacteria reduced Cd uptake and effected the rhizosphere microbial communities of lettuce. Journal of Hazardous Materials, 398.

\section{Figures}



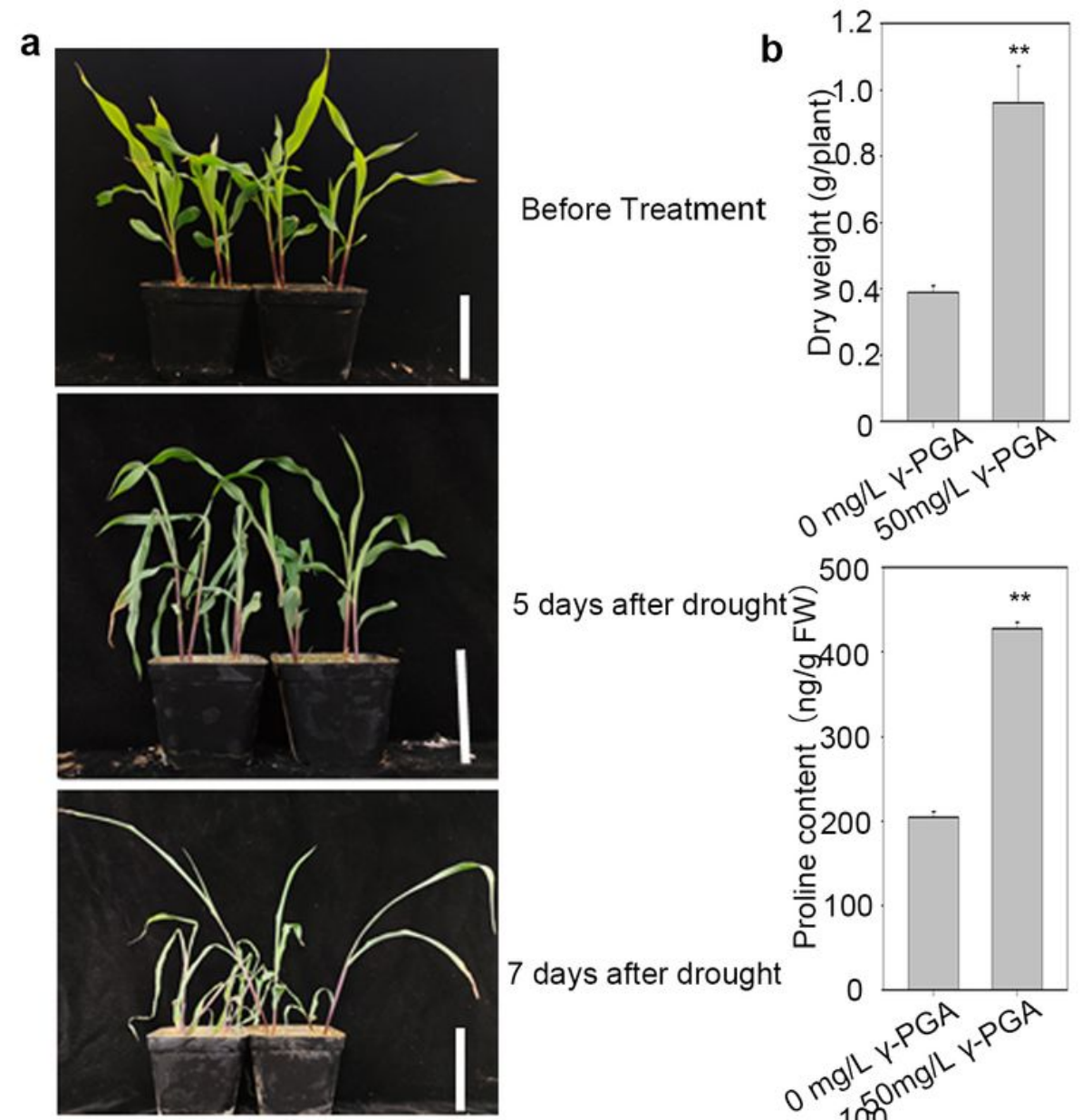

7 days after drought
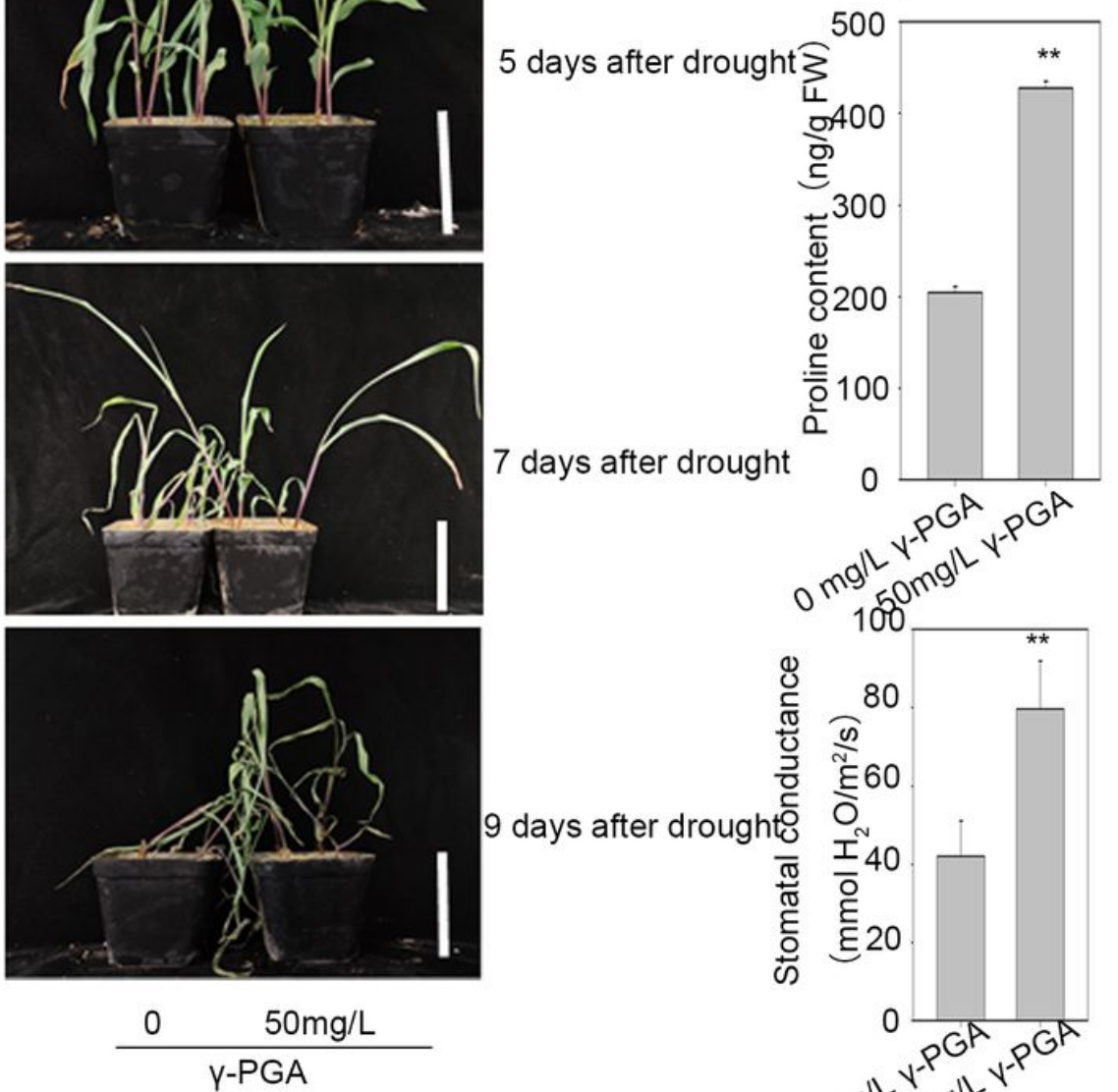

\begin{tabular}{lr}
0 & $50 \mathrm{mg} / \mathrm{L}$ \\
\hline & $\gamma-P G A$
\end{tabular}
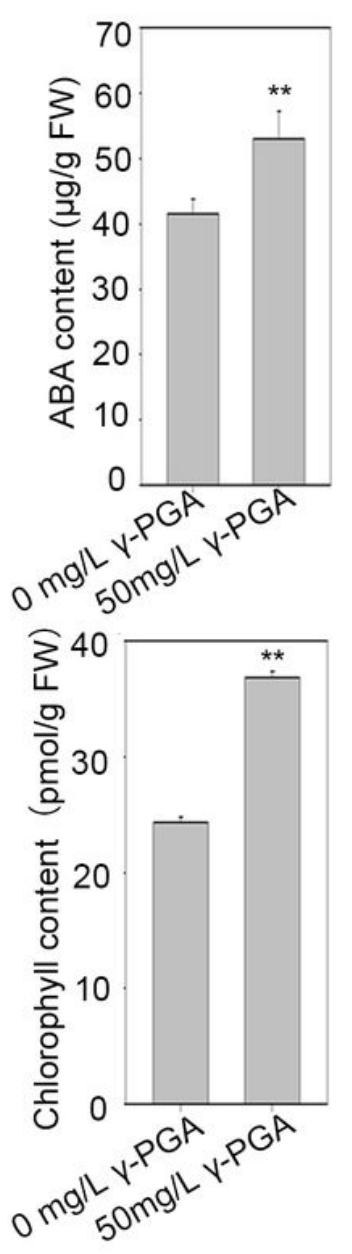
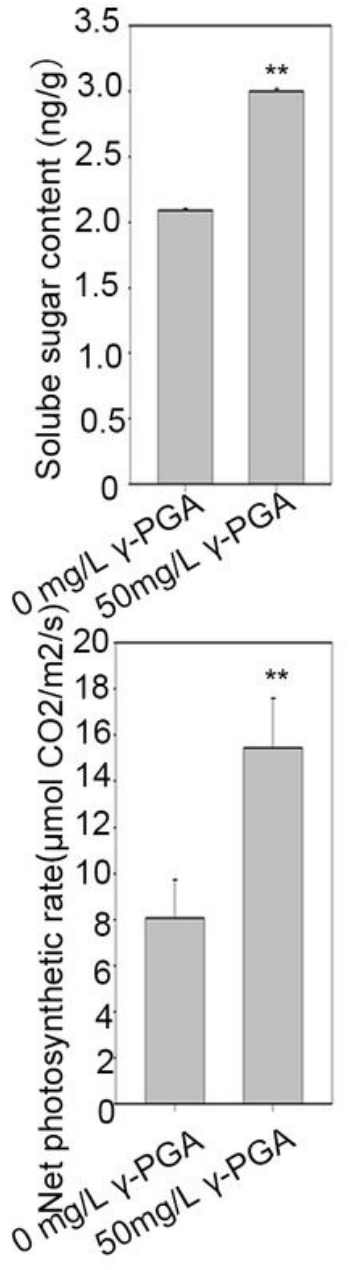

\section{Figure 1}

Phenotypes of maize added 50mg/L Y-PGA under drought stress, and the determination of related physiological indexes. (A) Phenotypes of maize added 50mg/L $y$-PGA under drought stress. (B) The determination of related physiological indexes (dry weight, contents of ABA, soluble sugar, proline, chlorophyll, net photosynthetic rate, and stomatal conductance) under drought stress for $5 \mathrm{~d}$. Values are means $\pm s d$ ( $n \geq 3$ repeats). Significant differences are indicated by asterisks ( $* \star, P \leq 0.01)$. 
a

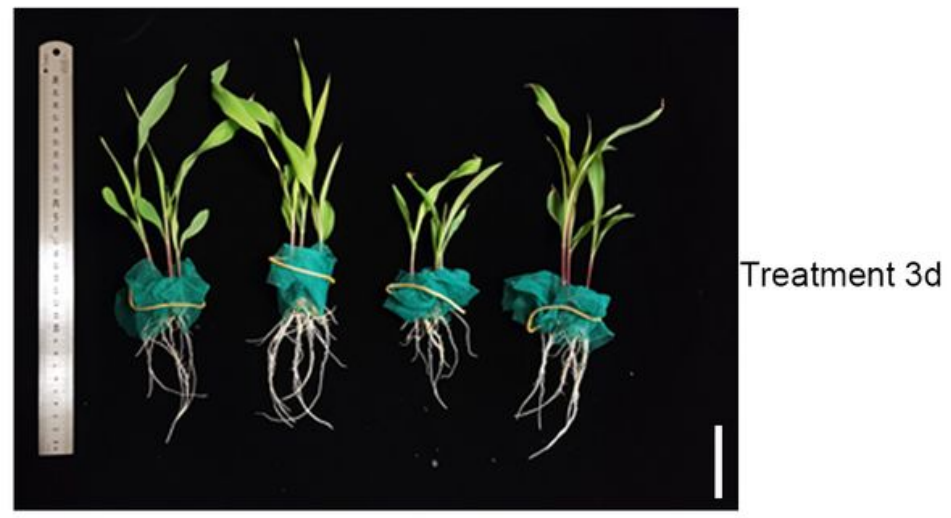

$\frac{\mathrm{CK} \mathrm{CK}+\gamma-\mathrm{PGA}}{\text { Control }} \frac{\mathrm{CK} \mathrm{CK}+\gamma-\mathrm{PGA}}{18 \% \text { PEG }}$

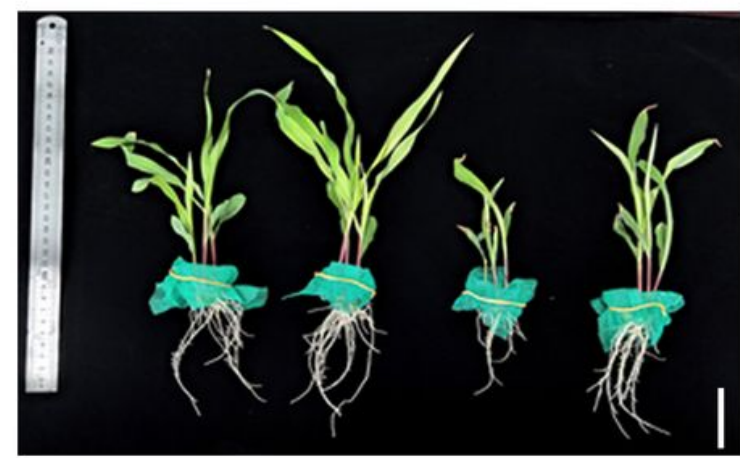

$\frac{C K C K+\gamma-P G A}{\text { Control }} \frac{C K C K+\gamma-P G A}{18 \% \text { PEG }}$
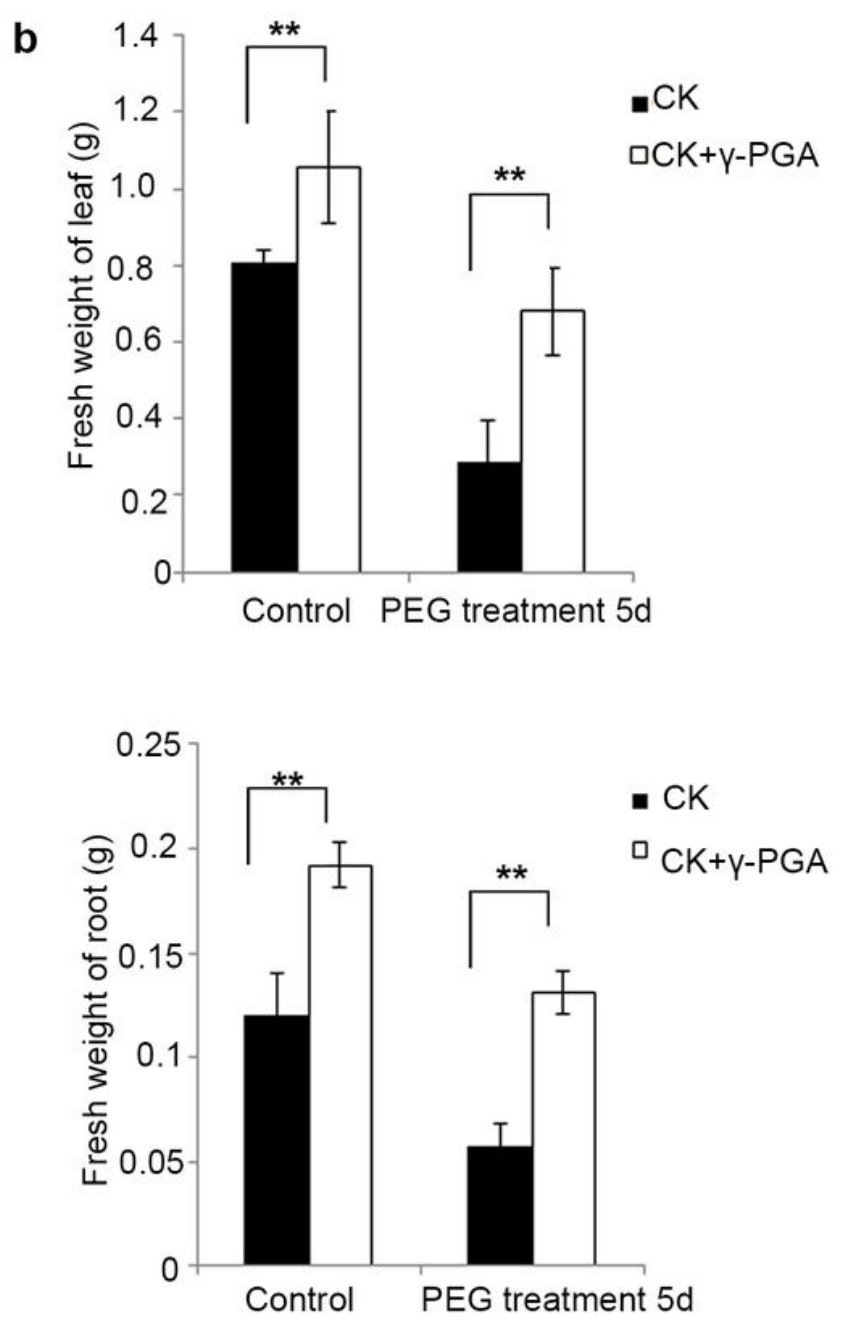

\section{Figure 2}

Phenotypes of maize added $\mathrm{Y}$-PGA under drought stress treatment with the $18 \%$ PEG6000 solution. (A) Phenotypes of maize added $\mathrm{Y}$-PGA (10KD, 50mg/L) under drought stress treatment with the $18 \%$ PEG6000 solution. (B) Fresh weight of the leaf and root of maize added $y-P G A$ and control maize under drought stress treatment for $5 \mathrm{~d}$ with the $18 \%$ PEG6000 solution. Values are means $\pm \mathrm{sd}$. Bars represent means $\pm s d$ ( $n \geq 3$ repeats). Significant differences are indicated by asterisks ( $\star *, P \leq 0.01)$. Bars $=5 \mathrm{~cm}$. 
a
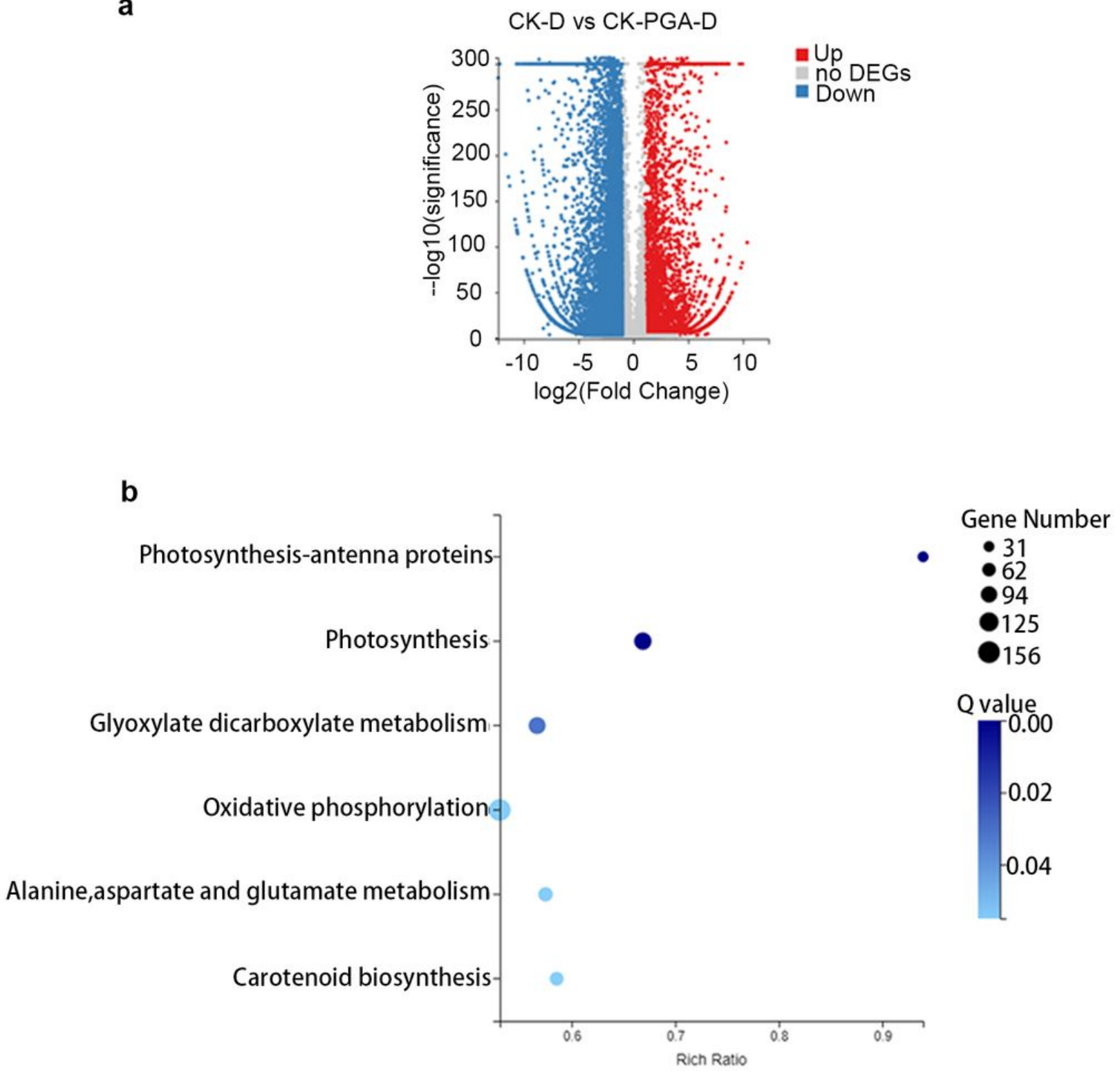

\section{Figure 3}

The differentially expressed genes (DEGs) identified by RNA sequence analysis and KEGG enrichment analysis of DEGs. (A) The number of differentially expressed genes (DEGs) identified by RNA sequence analysis. (B) The KEGG enrichment analysis of DEGs, Q value $\leq 0.05$. 


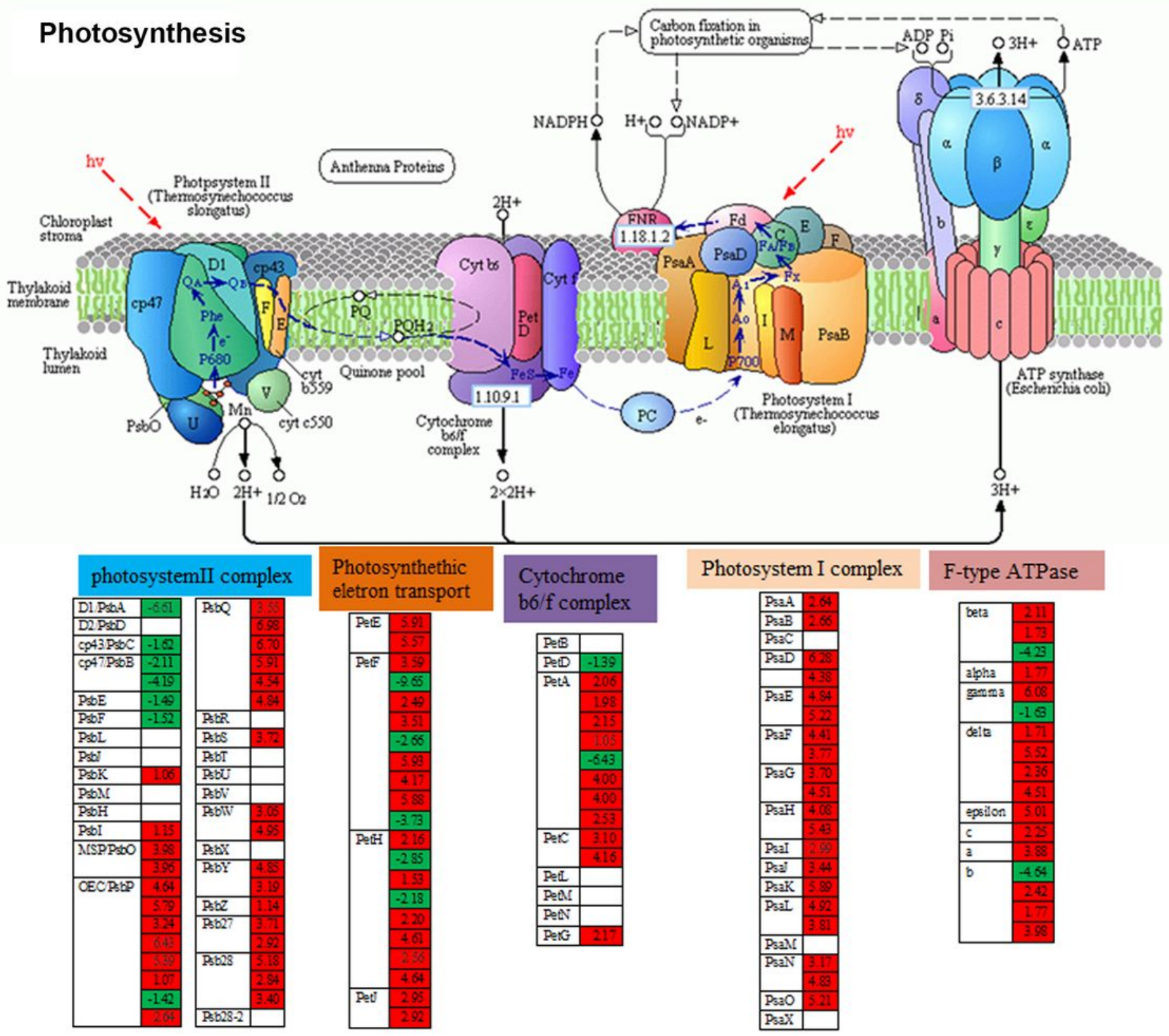

\section{Figure 4}

The DEGs involved in photosynthesis. Leaves from maize added $y-P G A$ under drought stress was collected for RNA sequencing. The absolute values of log2 (CK $+\gamma-P G A / C K) \geq 1$ and FDR $<0.001$ were used as the criteria for DEGs. The color of the box represented up (red) and down (green)-regulated (CK+ $\gamma-P G A / C K)$ genes, the value in the box was the log2 $(C K+\gamma-P G A / C K)$ of the genes in the leaves $\left(C K+\gamma^{-}\right.$ $\mathrm{PGA} / \mathrm{CK})$ under drought stress. The pattern of photosynthesis came from KEGG (http://www.genome.jp/kegg/). 
a

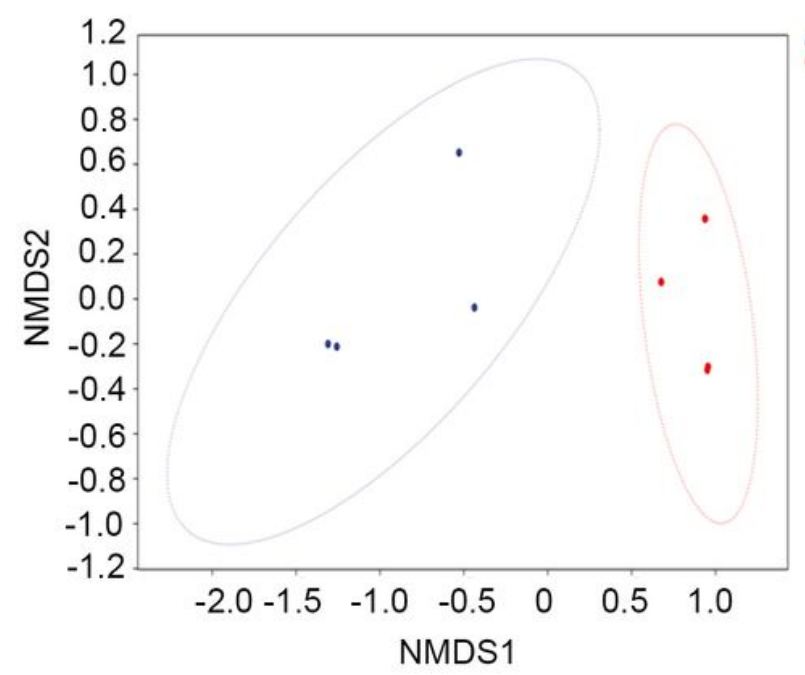

b 100

- CK-drought - CK-Y-PGA-drought

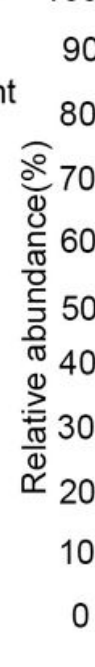

Proteobacteria - Actinobacteria - Chloroflexi Bacteroidetes Acidobacteria - Firmicutes - Verrucomicrobia - Patascibacteria Cyanobacteria Gemmatimonadetes ㅁ. Others

\section{C}

The current LDA threshold is 3.19

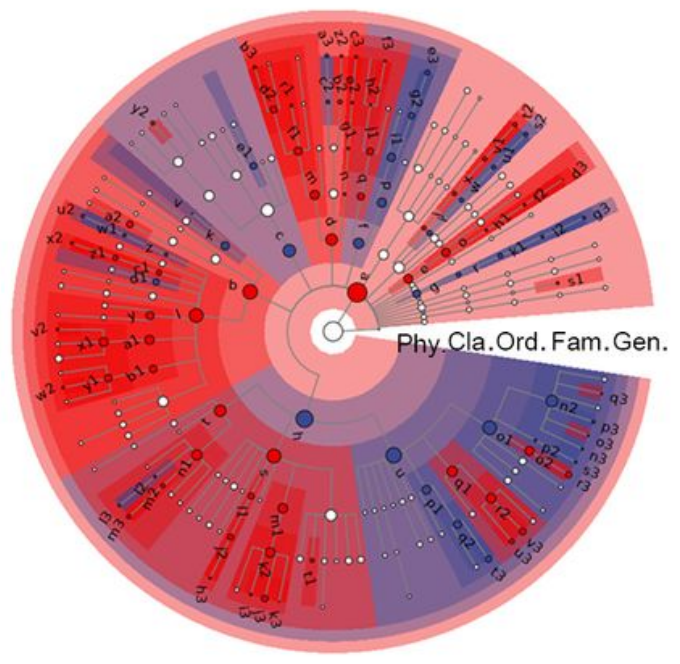

CK-drought

CK-y-PGA-drought

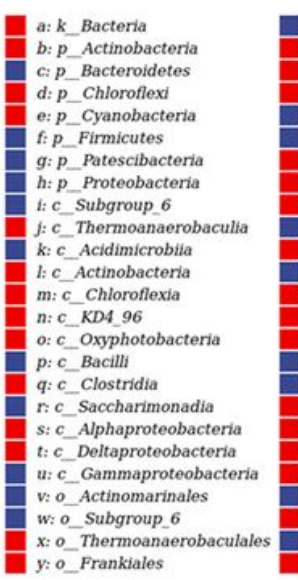

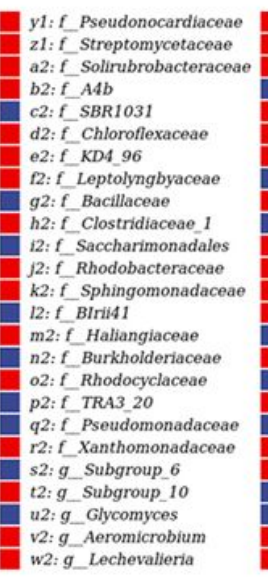

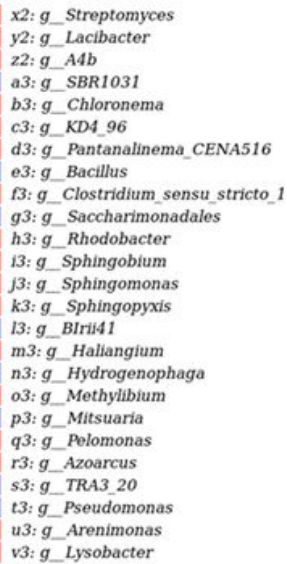

\section{Figure 5}

The NMDS, relative abundance and LEfSe analysis. (A) Non-metric multidimensional scaling (NMDS) showed the grouping patterns of the samples based on weighted UniFrac distance of all community. Each colored dot represented a sample. (B) The influence of $\mathrm{Y}$-PGA on the relative abundances of bacterial communities at phylum level in the rhizosphere soil of maize. c. LEfSe analysis (LDA $\geq 3$ ) showed the species with the most significant variation in the rhizosphere soil of control and $y$-PGA added maize under drought stress. 


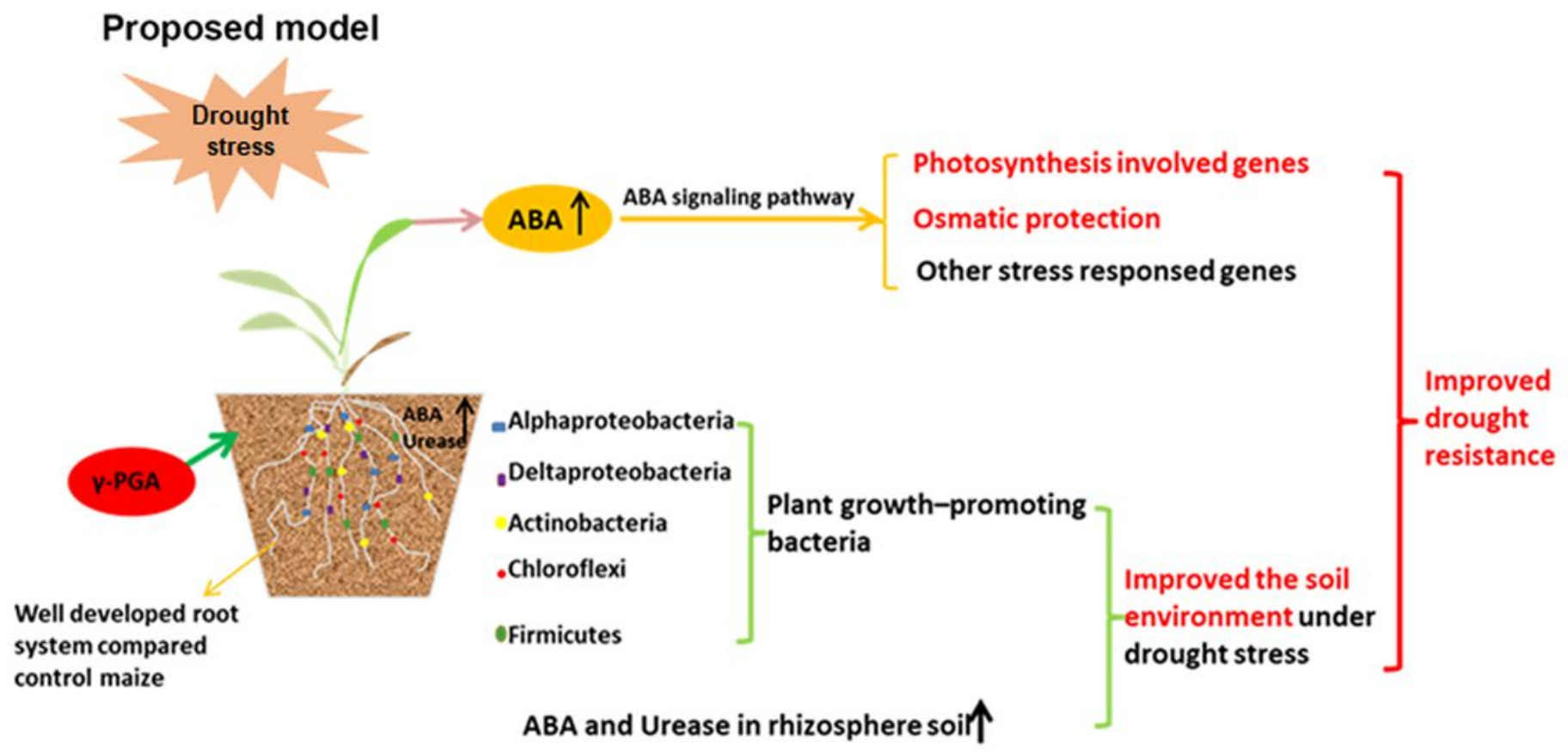

Figure 6

Proposed model for the role of $\mathrm{Y}$-PGA on maize under long-term drought. $\mathrm{Y}$-PGA can improve the drought resistance of maize by regulating the expression of $A B A$ biosynthesis, ABA signal transduction related genes, photosynthesis-related genes and other stress-responsive genes (osmatic protection, stress response and protein folding genes) and enriching the plant-promoting bacteria such as Actinobacteria, Chloroflexi, Firmicutes, Alphaproteobacteria and Deltaproteobacteria. In addition, ABA contents and urease activity in maize rizosphere soil were also increased.

\section{Supplementary Files}

This is a list of supplementary files associated with this preprint. Click to download.

- Supplementarymaterials.zip 